\title{
Pneumatic Driven Positioning and Alignment System for the Assembly of Hybrid Microsystems
}

\author{
Christian Brecher, Martin Freundt, and Christian Wenzel \\ Fraunhofer Institute for Production Technology, Steinbachstrasse 17, \\ 52074 Aachen, Germany, Tel.: +49(0)241-8904-253, Fax: +49(0)241-8904-6253 \\ martin.freundteipt. fraunhofer. de
}

\begin{abstract}
Micro assembly is typically characterized by positioning tolerances below a few micrometers. In the case of the assembly of hybrid micro systems, such as optical systems, micro ball lenses or micro probes for measurement tasks, even positioning accuracies in the sub-micrometer range have to be achieved. The efficiency of the use of automated handling devices is strongly influenced by the flexibility of the equipment and the required application specific customizations. In this context a high precision assembly head is presented. It upgrades conventional robots with the ability to do fine alignment steps in sub-micrometer resolution and 6 DOF. Therefore it is equipped with a universal endeffector structure.
\end{abstract}

Keywords: Optical assembly, Alignment, Micro Assembly, Micro Gripper, Assembly Head, Positioning, Air Bearing, Pneumatic Actuator, FlexibilityKeywords.

\section{Introduction}

Micro assembly is typically characterized by positioning tolerances below a few micrometers [1]. In the case of the assembly of hybrid micro systems, such as optical systems, micro ball lenses or micro probes for measurement tasks, even positioning accuracies in the sub-micrometer range have to be achieved [2].

The assembly is one of the most value creating process steps, as it allows to combine two materials or production processes in one product. Especially for hybrid micro systems, the assembly process step creates product functionality respectively a product value, larger than the total of the two assembled components had before. This merging of technological properties takes place within electro optical systems and other advanced technology focused applications.

Due to the need of highly accurate assembly systems and extensive alignment procedures, the assembly of high precision optics and micro systems is still characterized by customized solutions $[3,4]$. On behalf of this background, the Fraunhofer IPT has developed a new approach how to realize a highly flexible, fast and cost-efficient hybrid micro assembly processes. Main element of this approach is a robot guided assembly head, capable of adding high precision manipulation capabilities on conventional handling devices.

Within a handling process of the hybrid system, the robot guided active assembly head will be pre-positioned by a conventional handling device like an industrial robot. 
Thereby the large working area and high dynamics of the conventional system can be combined with the features of the assembly head, designed to compensate position and alignment errors with sub-micrometer accuracy. The manipulation precision required for the micro-assembly is therefore subsequently realized directly at the tip of the assembly head.

\subsection{Process Scenario and Requirements}

Regarding universal usability, which has to be a goal, designing an assembly head which is supposed to be used as a all purpose tool, adding precision alignment functionalities to a conventional robot system, following challenges have to be considered:

1. Universal endeffector: Design of an endeffector or endeffector system capable to be used for various part materials and shapes. Therefore it should be able to compensate for process or part tolerances.

2. 6 DOF fine alignment: A fine alignment stage capable of compensating the position deviation of a conventional robot in all, 6 degrees of freedom. It also has to be capable to allow for a certain additional high precision manipulation movement after the deviation compensation process.

3. Referencing Sensor system: A solution allowing the assembly head to be used not only within active guided assembly processes but be also an adequate tool for open loop positioning processes.

4. Ease of integration: The assembly head, containing and providing the precision specific functionalities to the assembly process has to be designed in a way that allows for standard integration procedures. Therefore the assembly head has to come with its own control so that it can be used as a plug and play device, only requiring power, digital control signals a compressed air supply.

These boundary conditions result in a process chain, described in figure 1 . Within this process the conventional robot is used for prepositioning with its specific dynamic and precision. Once the robot has reached its designated position, the assembly head starts with the high precision manipulation movement. In case of an open loop positioning process, the assembly head therefore acquires its position relative to a reference mark localized next to the working place with its integrated sensor system. In case of an active guided assembly and alignment process the assembly head can be utilized to directly adjust the part. For the handling and alignment of optics, especially in the field of the diode laser fabrication, the parts have to be positioned within increments of about $100 \mu \mathrm{m}$ respect angle manipulation increments in the range of

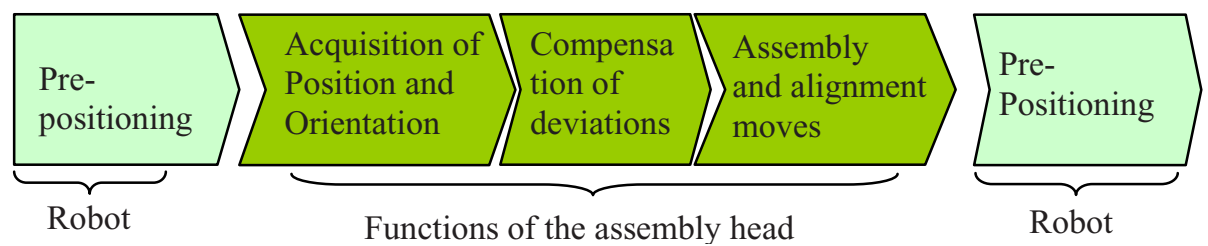

Fig. 1. Component handling process of a hybrid positioning device consisting of conventional robot and active assembly head 
Table 1. Essential requirements for a fine alignment system integrated into an assembly head for the realization of high precision manipulation

\begin{tabular}{ll} 
Function & Requirements \\
\hline Alignment & 6 DOF, $0.1 \mu \mathrm{m} / 0.001^{\circ}$ increments, $1 \mathrm{~mm} / 2^{\circ}$ travel range \\
Integration issues & $\begin{array}{l}\text { Max. workspace about } 150 \times 150 \times 150 \mathrm{~mm}^{3} \text {, cone shaped } \\
\text { outline for a good accessibility to the part, small mass, } \\
\text { robust about acceleration, miniaturized } \\
\text { Process }\end{array}$ \\
Flexible usable gripper tool, usable for various part shapes, \\
requirements & does not require endeffector exchange
\end{tabular}

$1 / 1000^{\circ}$. The typically actively controlled adjustment process requires alignment in up to $6 \mathrm{DOF}$ in order to allow for the product quality optimisation.

Concerning the combination of a conventional robot and an assembly head, as well requirements of the conventional robot system have to be considered. As conventional robots are not capable to reach the required precision demands, the drives of the conventional robot have to be deactivated in order to suppress control circuit vibrations. A survey of the main requirements is listed in Table 1.

In this context, an assembly head was developed which includes a 6 DOF fine alignment system, a universal endeffector and a referencing sensor system all controlled by an integrated control circuit allowing the use of the system as exchangeable gripping device, empowering conventional positioning devices to be applied for high precision assembly processes.

\section{Design of the Universal Usable Endeffector with Alignment Capabilities}

The design of the manipulation and alignment system has to meet the requirements of the endeffector working principle, as it is the system component carried by the rest of the system. Exchange and load scenarios, stiffness and precision requirements characterize the resulting requirements for the alignment system. The design challenge is to combine both functionalities within one device. Therefore, a concept of a hybrid endeffector and alignment structure was designed, allowing gripping and manipulating various materials and part shapes. The structure bases on three identical 3 DOF devices, each positioning one endeffector in $\mathrm{X}, \mathrm{Y}$ and $\mathrm{Z}$ direction, as shown in figure 2. As the distance between the three endeffectors towards each other has a significant influence on the manipulation range and resolution, the orientation of the $\mathrm{X}$-axis of all stages is radial to the centre of the structure as shown in figure 4 . As the main axis, it is driven by a combination of sequential controlled elliptec piezomotors, is capable to travel a significant distance of about $12 \mathrm{~mm}$. The second axis is orientated within tangential orientation, thereby. The third axes, in Z-direction allows for lifting and lowering the component as well as tilting the component around $\mathrm{X}$ and $\mathrm{Y}$-axes. Both, the second axes and the third are capable of travelling 1-2 mm depending selected resolution. 

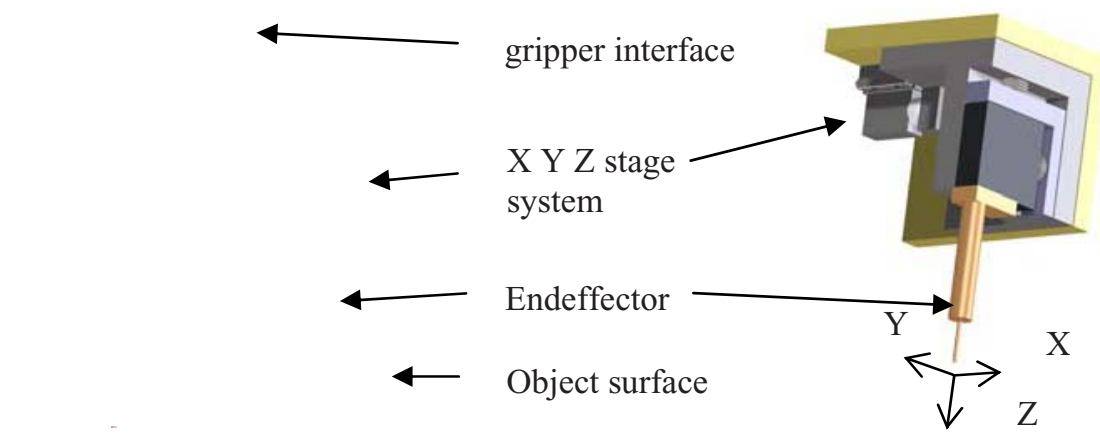

Fig. 2. Illustration of the function of a highly flexible and precise fine alignment device, system structure (left), function principle of the three axis stage (right)

Thereby following challenges within the design of robot guided, flexible and high precision assembly heads are addressed:

Flexible endeffector function. Gripping the component in three contact points creates a shape independent endeffector function. The three end effectors have to be designed in a way that they cannot apply any serious torque to the component. Thereby they define the position and orientation of the component and ensure a distinct handling process.

6D alignment functionality. Each of those end effectors, defining the position and orientation of the gripped component can be positioned within three translational degrees of freedom. With the adequate control system, any part shape can be manipulated in all degrees of freedom. The concept of three separate end effectors, allows to reduce the technical complexity. The design challenge of the fine alignment system can be reduced from a 6 DOF system towards a comparable simple 3 DOF system. This design concept is the key to be able to design a high precise and same time miniaturized manipulation device of six degrees of freedom.

Integration into an automation environment. The assembly head includes all required actuators, sensors and control devices, allowing the integration into existing robot systems without extensive customizing of the automation setup. It will have to be supplied with compressed air, power and the digital data for the execution of gripping and alignment tasks via a SPI-bus-interface. This ensures the usability of the system and allows a tool like use for various precision handling and alignment tasks.

\section{Design of an Endeffektor Manipulation Stage}

Due to the endeffector concept, the complexity of the design challenge is reduced to a $3 \mathrm{DOF}$ positioning system. The requirements onto the device concerning stiffness and load capacity are also minimized, as only three axes have to be realized. Further, the components, going to be manipulated, are lightweight compared to required stage structures. An additional load introduced by a complex endeffector can also be spared. 
Concerning high precision requirements, all guidings are based on air bearings. Those air bearings are integrated within the structure of the slides due to construction space and lever minimisation. The three required 3 DOF positioning devices are equipped with miniaturized air bearings to ensure absolute precision capabilities. Its main slide is driven by a pattern of six elliptic piezo motors allowing to archive long travel ranges of more than $10 \mathrm{~mm}$. For high precision movements, an optical sensor system is integrated within the main stage, allowing to measure the primary slide position. Slide two and three are integrated within the structure to optimise the load profile on the air bearings and reduce the size of the stage system. Figure 3 illustrates the integral design. In order to avoid disturbing forces caused by tubings for the supply of the air bearings and wiring for actuators, a system of contact free transmissions for compressed air is integrated within the air bearing gabs. (patent application DE 10 2007023516 A1).

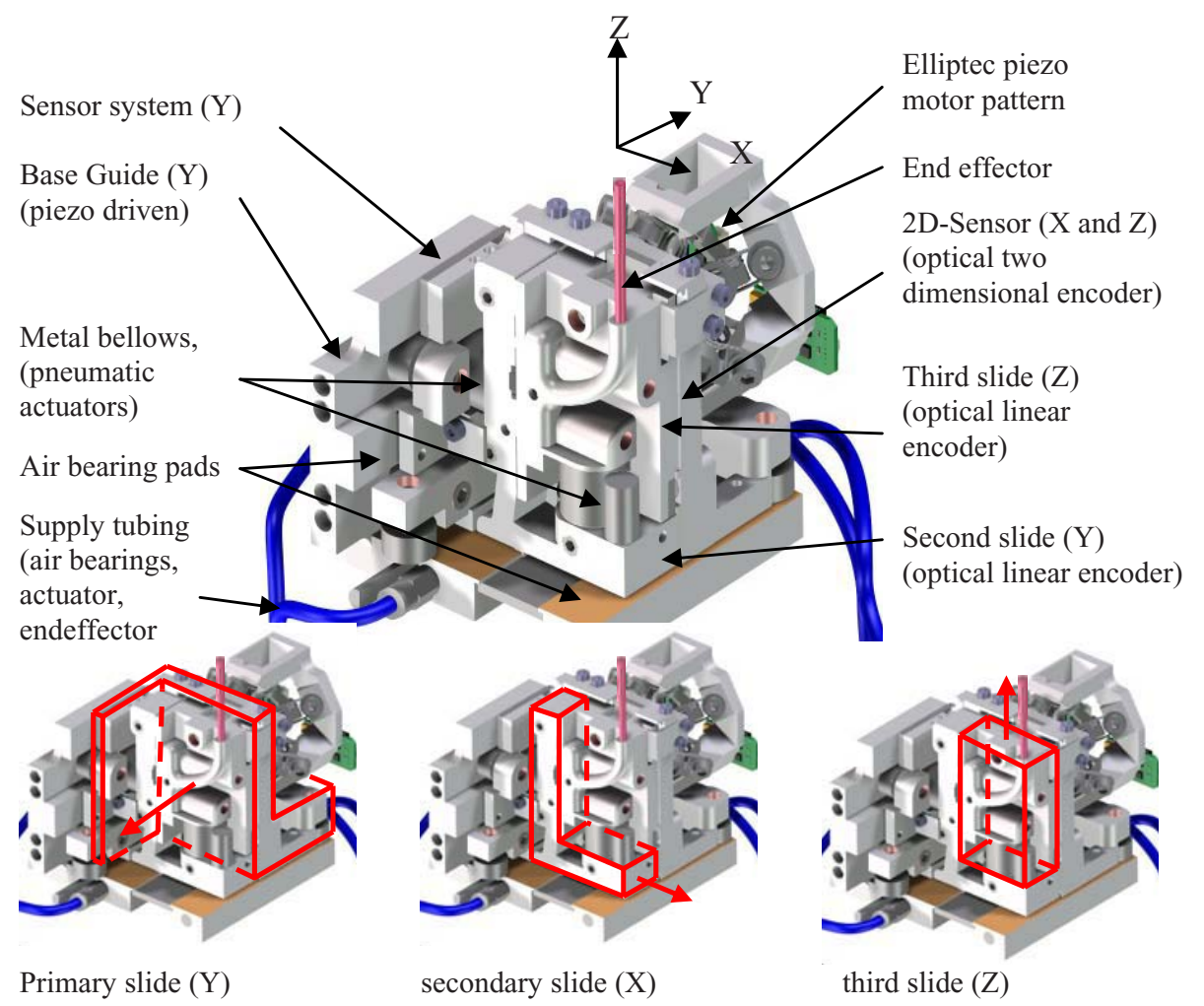

Fig. 3. CAD-Model of the three axis, air bearing stage with internal transmission of compressed air and pneumatic driven second and third axis

They allow for supplying of the stages and for providing the compressed air signal to the metal bellows actuators, driving the secondary and third stage. A 2D planar encoder device integrated on the first slide detects the position of the second and third 
slide. It allows for the accurate measurement and control of the second and third axis, which are driven by the comparable weak but extremely compact metal bellow actuators. Due to the complex air bearing structure and the tight tolerances within air gab, all air bearings are designed preloaded by adjustable magnets. This adjustment opportunity of the preload enables to put the complex air bearing design into operation. Due to the required design with several air bearing pads, integrated within the slides all slides are fabricated using laser sintering technology allowing to route free form boreholes and cavities through the slides and thereby enable to supply each single air bearing pad, made out of porous graphite. All air bearing surfaces are ultra precision diamond milled allowing for small bearing gabs and a precise alignment of the slides towards each other.
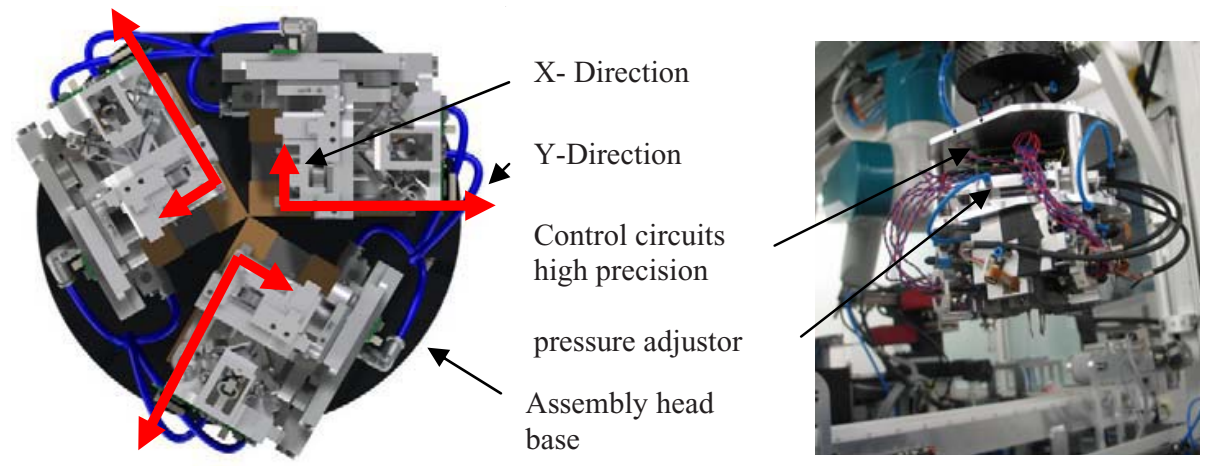

Fig. 4. Illustration of the orientation of the endeffector positioning capabilities (bottom view, left) and a photo of the assembly head prototype within a robot system (right)

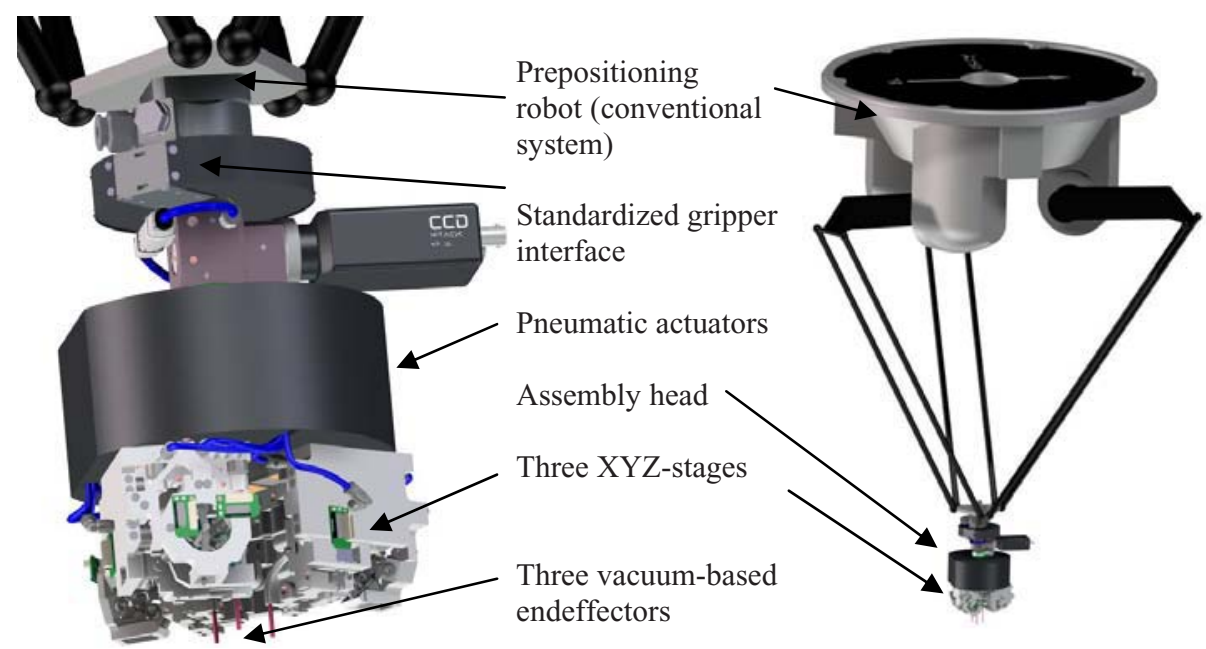

Fig. 5. CAD-Model of the assembly head based on three identical XYZ-stages each positioning a vacuum based, leakage afflicted endeffector 
The three stages are oriented under 120 degree. The main slide thereby is orientated in radial direction allowing an adaption of the end effector configuration. In order to adapt to component shape and size, the distance of the end effectors can be modified from $0.2 \mathrm{~mm}$ to about $12 \mathrm{~mm}$. The assembly head is equipped with an on board electronic including sensor evaluation circuits, microprocessor based control unit and driver circuits for the elliptic piezomotors. The whole assembly head thereby requires compressed air to run the air bearing and actuators, $24 \mathrm{~V}$ power. The communication with a superior process control is avaible via RS232 or SPI. This ensures that the assembly head can be used as an all-purpose tool within conventional robots. Figure 5 illustrates the integration within a robot system at the Fraunhofer IPT. With this assembly head design conventional robots will be usable for precision assembly processes. In case of objects which can not actively aligned in within a process, a referencing sensor device is required. In order to avoid part specific adaption work within process implementation, it is required to apply a universal sensor system. The sensor system has to be able to be integrated within the assembly head structure allowing using it in multiple positions within the assembly area.

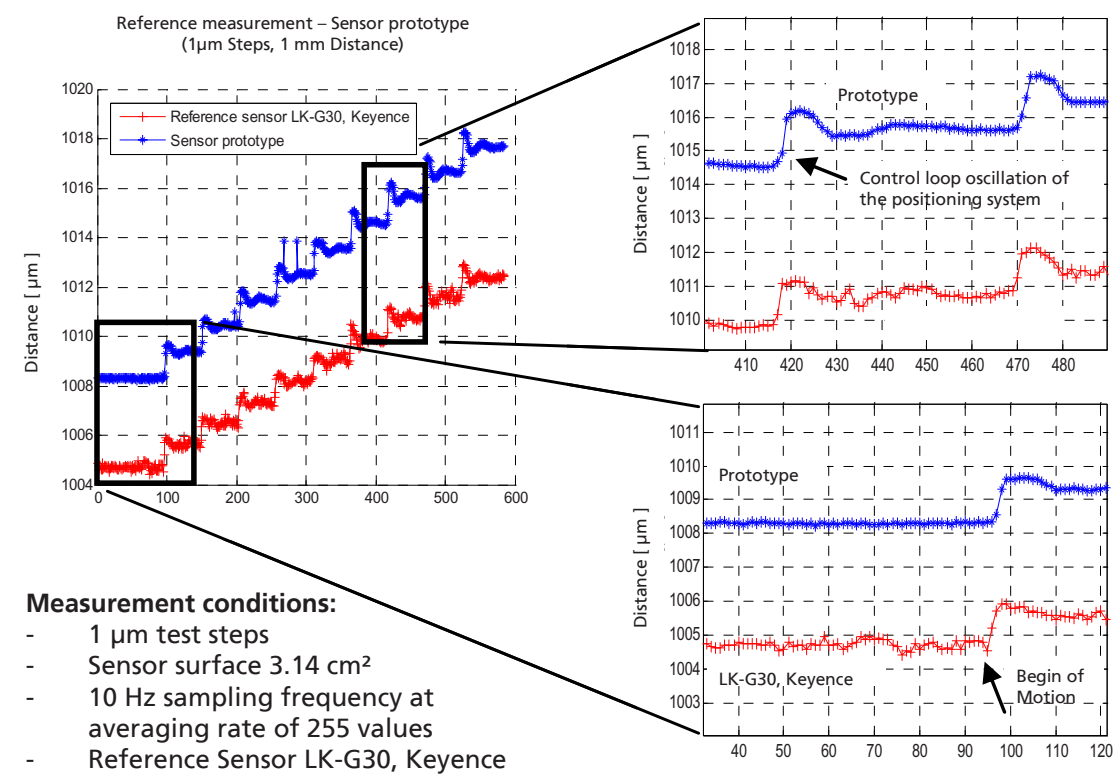

Fig. 6. Preliminary test results of a capacitive sensor element with sensor head integrated digital readout, designed for the setup of a 6 DOF robot guided referencing sensor system

Commercially one-dimensional sensor systems with adequate resolution in the sub micrometer range and even measurement range of more than $1 \mathrm{~mm}$ are avaible. In order to combine six sensors, allowing to measure the position and orientation deviation of the robot system, the signal analysis circuits have to be integrated within the assembly head. Due to the boundary conditions, especially the application within conventional robots, only digital signals can be failure-free transferred through a 
gripper interface and robot wiring. In behalf of this challenge a prototype of a compact capacitive sensor element with onboard signal digitalization was designed.

Preliminary testing of noise and resolution allows to expect detection resolution of about $100 \mathrm{~nm}$. Figure 6 presents preliminary test results of a prototype sensor element which has a build in digital readout using SPI communication.

\section{Outlook}

The technology of gripping and manipulation components based on three vacuum based endeffectors presented here was implemented within a prototype assembly head. It is going to be put into operation and will be tested within its handling precision and usability. Additional research will be conducted within the field of sensor integration in order to be able to archive adaptivity and allow for the design of semi automated process programming.

\section{Acknowledgements}

The authors would like to thank the »Deutschen Forschungsgemeinschaft (DFG)« for supporting the »Greifer und Montagemaschinen« research project as part of the special research topic SFB $440 »$ Assembly of hybrid micro systems «.

\section{References}

1. Heuer, K., Hesselbach, J., Berndt, M., Tutsch, R.: Sensorgeführtes Montagesystem für die Mikromontage. In: Robotik VDI-Berichte Nr. 1841, München, June 17-18, pp. 39-46 (2004) ISBN 3-18-091841-1

2. Brand, U., Wilkening, G.: Dimensionelle Messtechnik und Normung für die Mikrosystemtechnik - Eine Frage des Maßstabs. In Mikroproduktion 3/2006

3. Raatz, A., Hesselbach, J.: High-Precision Robots and Micro Assembly. In: Proceedings CoMa (2007)

4. Peschke, C.: Mehr-Achs-Mikrogreifer zur Handhabung biegeschlaffer Mikrobauteile, Dissertation, RWTH Aachen (2007) 